\title{
GLUTATHIONE CONTENT AND GLUTATHIONE PEROXIDASE ACTIVITY OF SPERM IN MALES WITH UNEXPLAINED INFERTILITY
}

\section{Danail I. Martinov, \\ Nina P. Ayvazova ${ }^{2}$, Emiliana I. Konova ${ }^{1,2}$, Milena A. Atanasova}

Department of Anatomy, Histology, Cytology and Biology, Medical University - Pleven

${ }^{1}$ Reproductive Health Center, Medical University - Pleven ${ }^{2} M C$ Clinical Institute for Reproductive Medicine - Pleven

\author{
Corresponding Author: \\ Danail I. Martinov \\ Biology Division, \\ Medical University Pleven \\ 1, Kliment Ohridski Str. \\ Pleven, 5800 \\ Bulgaria \\ e-mail: danail_martinov@abv.bg
}

Received: December 04, 2020

Revision received: February 19, 2021

Accepted: May 27, 2021

\section{Summary}

The study aimed to investigate glutathione peroxidase (GPx) activity and glutathione (GSH) levels in the sperm of patients with unexplained infertility. The sperm samples were collected from subjects with normal semen parameters divided into fertile and infertile groups. Sperm analysis was performed according to the $2010 \mathrm{WHO}$ criteria. Measurement of the GPx activity and GSH were performed by enzymatic assay kits. The higher enzymatic activity recorded in spermatozoa and seminal plasma in the infertile group was close to the significant one $-\mathrm{p}=0.054$ for seminal plasma andp $=0.086$ for the spermatozoa.GSH levels were higher in the fertile group in the seminal plasma $(\mathrm{p}=0.045)$. Defining the causes of unexplained infertility requires the addition of oxidative stress. In patients with unexplained infertility, the level of glutathione is reduced, and the activity of one of the significant enzyme antioxidants GPx is not changed significantly and even shows a tendency to rise.

Keywords: male infertility, oxidative stress, glutathione peroxidase, glutathione

\section{Introduction}

Infertility is a condition in which no pregnancy is achieved after regular and unprotected sexual intercourse for 12 months [1]. According to the official statistics, the number of childless couples in Bulgaria is about 270000 . This means that almost every sixth couple is affected. Such couples resort to assisted reproductive technologies (ART). Unfortunately, the success of ART is relatively low - about $30 \%$. One of the reasons for infertility and the low success rate of in vitro procedures is oxidative stress (OS).

The free radicals have a substantial positive effect on spermatogenesis. They change the proliferation, differentiation, and function of the cells $[2,3]$. Nevertheless, they also have a negative effect. If the balance between free radicals and the antioxidative system is disturbed, reactive oxygen species (ROS) can and harm cell structures. ROS reactivity varies widely and is affected by temperature and molecule concentration. Under normal physiological conditions, ROS are constantly generated in the organism. Those radicals are eliminated by the antioxidative protective system [4]. However, in various pathological 
conditions, their generation can intensify, and the antioxidative capacity of the biological system can prove insufficient to eliminate their production. As a result, they can attack and harm all cell structures [4]. The condition of free radicals hyperproduction andlor lowered antioxidative protection is defined as oxidative stress.

The pathological effect of the activity of the free radicals in the male reproductive tract is connected with DNA fragmentation, lipid peroxidation, lowered antioxidant capacity of the seminal plasma, and apoptosis. This leads to reduced fertility. It can also be a reason for miscarriages and no pregnancies [5].

When the reason for infertility is not defined, the patients are categorized as patients with unexplained infertility. They can be put in two groups - idiopathic infertility and unexplained male infertility. According to Gudeloglu A, Brahmbhatt, men with idiopathic infertility are three times more than those with unexplained infertility (33\% against $11 \%$ ) [6]. Normal results after a physical examination and endocrine testing are seen in idiopathic infertility. However, the semen quality is lower than that in patients with unexplained infertility who show normal levels of semen analysis results [7]. Unexplained infertility is a diagnosis made in patients who, despite normal levels of conventional semen analysis, standard history, and physical examination and exclusion of the female factor (fertile partners), cannot achieve pregnancy with regular unprotected contacts for 12 months. The possible factors that hinder conception are anti-sperm antibodies, DNA fragmentation, higher level of free radicals, sperm dysfunction, disturbed sexual function (erectile dysfunction) [6].

Despite the direct connection between male infertility and disabilities caused by oxidative stress, the research in this field is often underrated. The treatment should be directed at restoring the balance of the oxidant/antioxidant system. Instead, such patients go for in vitro fertilization (IVF), intracytoplasmic sperm injection (ICSI), or intrauterine insemination (IUI). According to different researches, 40 to 80 $\%$ of subfertile men have high levels of ROS [8].

Both the leukocytes and spermatozoa in each ejaculate are the source of free radicals (oxidative stress). The ability of spermatozoa to produce ROS is inversely proportional to their maturity. Immature spermatozoa contain a small quantity of residual cytoplasm in their middle part, rich in glucose-6-phosphate dehydrogenase - an enzyme that controls the glucose levels and catalyzes the production of NADPH through the hexose monophosphate shunt. NADPH is a substrate of NADPH-oxidase (NOX) - an enzyme in the spermatozoon membrane. That is why men with teratozoospermia have higher levels of ROS compared to normozoospermic men [9].

Human spermatozoa are highly susceptible to lipid peroxidation because their plasma membranes are rich in polyunsaturated fatty acids (PUFA). PUFA increases membrane fluidity, which is essential for the fusion of gamete membranes in the process of fertilization [10].

On the other hand, mature spermatozoa with a minimal quantity of cytoplasm and insufficient protection by the cytoplasm antioxidants are susceptible to potential oxidants. Seminal plasma antioxidants protect them through enzymatic and non-enzymatic mechanisms. Similar to all cells, spermatozoa have an antioxidant protective system. Mature spermatozoa have low intracellular antioxidant capacity due to the minimal quantity of cytoplasm. The intracellular enzymatic protection against ROS consists of superoxide dismutase (SOD), nuclear glutathione peroxidase (GPx), peroxiredoxin (PRDX), thioredoxin (TRX), and thioredoxin reductase (TRD) [11].

Glutathione peroxidases catalyze the reduction of hydroperoxides using $\mathrm{GSH}$ as a reductor. They are a family of 8 enzymes that act in cells located in different tissues. Their leading role is to protect the cells from oxidative impairment [12]. All GPx enzymes are tetramers built by identical monomers, except for the phospholipid hydroperoxide GPx (GPx4), which is a monomer. Each monomer contains selenocysteine in the active core, which directly participates in the two-electron reduction of the peroxide substrate. The reduced glutathione GSH serves as a source of electrons. GPx4 and GPx 5 are mostly expressed in the male reproductive tract [13].

Glutathione can react with radicals and electrophiles. It can also be the source of electrons for Glutathione peroxidase. The 
glutathione (L-glutamyl-L-cysteinyl glycine, GSH) is a tripeptide, very common in plants and animals. It plays a crucial role in many biological processes. It is a part of protein and nucleic acids synthesis and molecular transport. However, its most significant role is in protection against the oxidative effect of the free radicals. It serves as the nucleophile substrate of the glutathione transferase in xenobiotic detoxification. It is also a donor of electrons to the glutathione peroxidase family enzymes in the reduction of $\mathrm{H}_{2} \mathrm{O}_{2}$. Glutathione also takes part in transporting the amino acids and maintaining the protein sulfhydryl reduction status. Its concentration varies between several micromoles in the blood plasma and several millimoles in tissues (liver). The concentration of glutathione is different in different types of tissues in the male reproductive tract. A significant quantity of glutathione has been found in the testes, in secretions of the glands in the reproductive tract, and in the spermatozoa, isolated from the epididymis. Glutathione is found intracellularly in the spermatozoa and the seminal plasma [14].

ROSarenecessary fortheprocess of conception since they take part in the hyperactivation, motility, capacitation, and acrosome reaction [15]. Therefore, a delicate balance should be maintained between the physiological optimum and hyperproduction. If ROS level is too high, the antioxidative protection system can be overloaded or even induce its upregulation and thus achieve an adverse effect, i.e., lead to levels lower than normal.

The aim of our study was to investigate glutathione peroxidase activity and the levels of its substrate glutathione (GSH) in sperm samples from patients with unexplained infertility.

\section{Materials and Methods}

\section{Patients}

The research was conducted from June 2017 to September 2018. It included two groups controls and patients. They were selected from patients of the Medical Center of St. Elisaveta Clinical Institute for Reproductive Medicine in Pleven. All the patients signed informed consent forms. Volunteer recruitment, sperm sample processing, and semen analysis were conducted at the Medical Centre of the Clinical Institute for Reproductive Medicine - Pleven. The measurements of glutathione and glutathione peroxidase were conducted at the Medical University - Pleven. The research was approved by the Ethics Commission of MU-Pleven.

The control group included fertile men with data for normozoospermia $(n=23)$ aged $34.6 \pm 6.2$ (age range 22-44). The controls had achieved pregnancy with their partners by having regular and unprotected sexual contacts for a calendar year.

The patient's group included men with unexplained infertility $(\mathrm{n}=24)$ aged $35.1 \pm 5.9$ (age range 29-46). These had not achieved pregnancy with their partners by having regular and unprotected sexual contacts for a calendar year and had conventional semen analysis had proved normal parameters (normozoospermia).

All volunteers were physically examined and underwent laboratory tests. Excluded from the research were men with conditions such as varicocele, cryptorchidism, and endocrine disorders, chronic diseases and urogenital infections, previous testicular traumas (absence of anti-sperm antibodies), as well as men who had been febrile for the last three months, and those with leukocyte concentration $>1.10^{6} / \mathrm{ml}$ ejaculate. Volunteers who had had a three-month antioxidant therapy were also excluded.

All men that took part in the research had normal sperm concentration and sperm motility according to the WHO standards from 2010. To eliminate the female factor, the female partners of the tested volunteers were with normal gynecological status and no endocrinopathies, and normal fertilization potential.

\section{Sample processing}

Sperm samples from the two groups were collected after 3 to 5 days of sexual abstinence and no alcohol intake. The material was extracted through masturbation, placed in nonpyrogenic containers, and then each sample was divided into two parts. After that, the spermatozoa and seminal plasma were separated through double density gradient centrifugation. Half of the thus separated samples underwent conventional analysis of concentration, motility, and morphology of the spermatozoa.

\section{Semen analysis}

After liquefaction, the sperm samples underwent 
conventional analysis following the WHO standards from 2010. The physicochemical parameters we assessed were: volume, viscosity, $\mathrm{pH}$, and ejaculate color.

Volunteers with a sperm concentration of $15 \times 10^{6} / \mathrm{ml}$, progressive motility $(\mathrm{a}+\mathrm{b} \%)$ of $32 \%$, and normal morphology were defined as normozoospermic.

The other half of the samples were frozen at $-80^{\circ} \mathrm{C}$. The spermatozoa were processed with cryoprotectant SpermFreeze (FertiPro) and frozen at $-196^{\circ} \mathrm{C}$. Before the glutathione and glutathione peroxidase analysis, the spermatozoa underwent sonification for membrane structure destruction and disposal of compartmentalization components.

\section{Gradient centrifugation}

The ejaculate processing was conducted through density gradient centrifugation. Two $\mathrm{ml}$ of sperm liquid was layered in a conic test tube in $90 \%$ SupraSperm gel (Cooper surgical), which had been layered with $45 \%$ SupraSperm gel in a 1:2 ratio. The samples were centrifuged for 16 minutes at $1600 \mathrm{rpm}$. After that, the supernatant was discarded, the residue was resuspended in a $1 \mathrm{ml}$ Sperm preparation medium (Cooper surgical) and centrifuged again for 7 minutes at $1400 \mathrm{rpm}$.

\section{Glutathione measurement}

The levels of glutathione were measured with a Cayman Chemicals kit (cat. No 703002). The kit uses GSH-reductase for the quantitative assessment of GSH. The measurement was conducted according to the manufacturer's instructions. We mixed $50 \mu \mathrm{l}$ of the sample with a $150 \mu \mathrm{l}$ cocktail containing a buffer, a cofactor, a mixture of glutathione reductase and glucose6-phosphate dehydrogenase, and 5,5'-dithiobis2-nitrobenzoic acid. After 25 minutes, the absorption was measured at a wavelength of 405 nm (Mithras LB 943 Multimode Microplate Reader). Each sample was tested two times, and the average glutathione concentration was calculated in $\mu \mathrm{M}$ by the standards.

\section{Glutathione peroxidase measurement}

Cayman Chemicals (cat. No 703102) kit was used. The kit indirectly measures GPx by conjugated reaction with glutathione reductase.
The measurement was conducted according to the manufacturer's instructions. We mixed $20 \mu \mathrm{l}$ of the sample with $50 \mu \mathrm{l}$ cosubstrate (NADPH). The reaction started with $20 \mu 1$ cumene hydroperoxide. The absorption at 340 nm wavelength was measured five times per minute (Mithras LB 943 Multimode Microplate Reader). Each sample was tested two times, and the average activity of the glutathione peroxidase in $\mathrm{nmol} / \mathrm{min} / \mathrm{ml}$ was calculated as the function of the absorption change $(\Delta \mathrm{A})$ per minute, as indicated in the manufacturer's instructions.

\section{Data analysis}

The statistical processing of the results was made with the statistical package SPSS19 of IBM Corporation. According to the ShapiroWilk test, the characteristics of the two groups were asymmetrically spread $(p<0.05)$. The Mann -Whitney test was used for the comparison of the characteristics. We used Levene's test for homogeneity regarding parameters such as days of abstinence, age, motility, and morphology so that volunteers were matched to the patients studied.

Results with a significant coefficient $\mathrm{p}<0.05$ were considered statistically reliable.

\section{Results}

\section{Conventional semen analysis}

The subjects in the groups did not differ by age and abstinence period and by parameters defined through conventional semen analysis (concentration, motility, morphology, and standard percentage of spermatozoa).

Table 1 presents the characteristics of the subject groups as in all cases, no significant differences between the two groups were found $(p>0.05)$. There was a positive correlation between the characteristics for motility and morphology $(r=0.628 * /$ Spearman) (Figure 1).

\section{Glutathione}

The data were asymmetrically spread. Significantly higher levels of glutathione in the seminal plasma in the fertile group were discovered (average value $2.1 \pm 0.27 \mu \mathrm{M}$ ) in comparison with the infertile group (average value $1,8 \pm 0,14 \mu \mathrm{M})(\mathrm{p}=0.045)$. Concerning the intracellular GSH, the discovered differences 
Table 1. Table 1 presents the characteristics of the subject groups as in all cases. No significant differences between the two groups have been spotted, i.e., $\mathrm{p}>0.05$. A positive correlation between the characteristics for motility and morphology were discovered $(\mathrm{r}=0.628 * /$ Spearman $)$

\begin{tabular}{cccc}
\hline Parameter (mean) & Control group $(\mathrm{n}=23)$ & $\begin{array}{c}\text { Unexplained infertility } \\
(\mathrm{n}=24)\end{array}$ & Difference $(\mathrm{p})$ \\
\hline Age (years) & $34.6 \pm 6.2$ & $35.1 \pm 5.9$ & $>0.05$ \\
\hline $\begin{array}{c}\text { Sperm concentration } \\
\text { (mill/ml) }\end{array}$ & $75 \pm 3$ & $72 \pm 5$ & $>0.05$ \\
\hline $\begin{array}{c}\text { Progressive motility } \\
(\mathrm{a}+\mathrm{b} \%)\end{array}$ & $44 \pm 2$ & $42 \pm 4$ & $>0.05$ \\
\hline Normal morphology & $4 \pm 1$ & $4 \pm 2$ & $>0.05$ \\
\hline $\begin{array}{c}\text { Teratozoospermic index } \\
\text { (TZI) }\end{array}$ & 1.35 & 1.35 & $>0.05$ \\
\hline
\end{tabular}

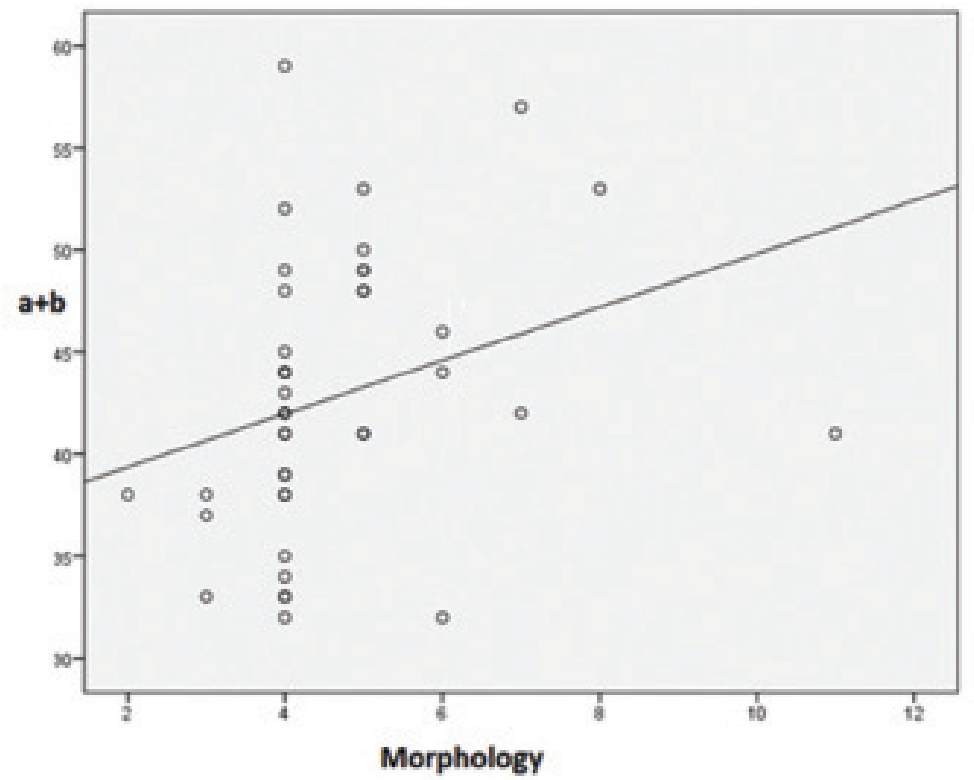

Figure 1. Positive correlation between motility and morphology of spermatozoa $\left(\mathrm{r}=0.628^{*}\right)$
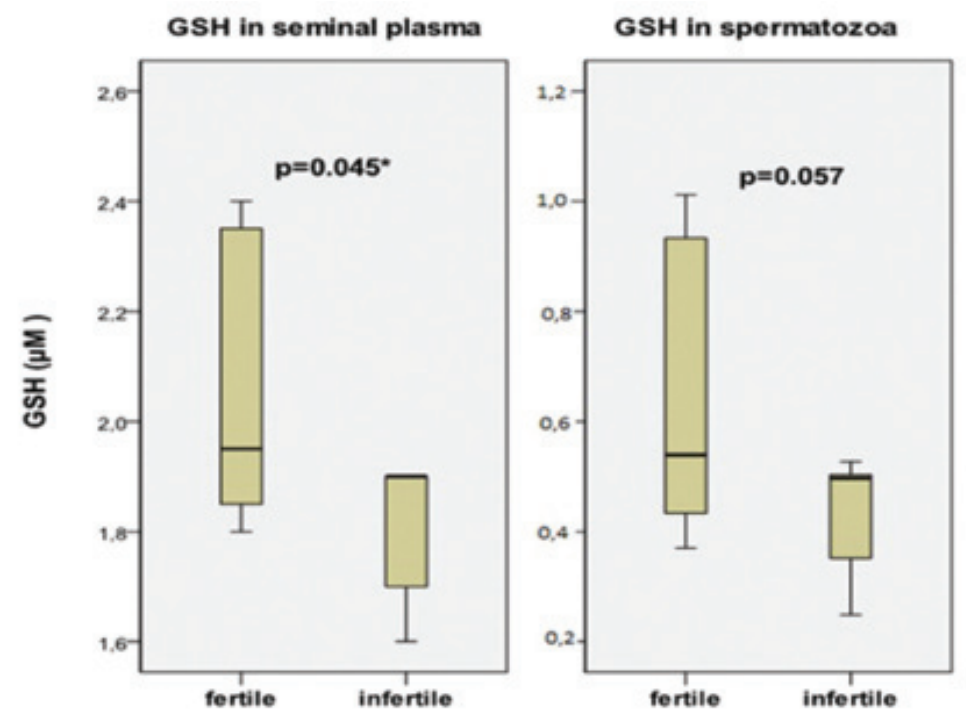

Figure 2. Levels of glutathione $(\mu \mathrm{M})$ in seminal plasma and spermatozoa of fertile and infertile men 


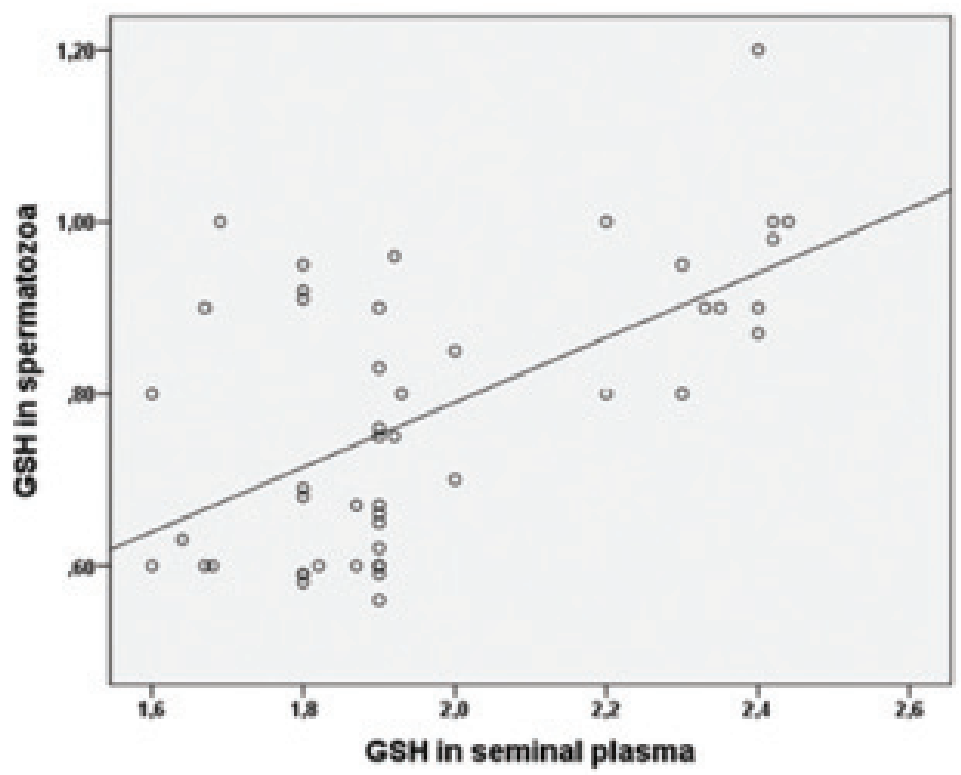

Figure 3. Positive correlation between the levels of GSH in the seminal plasma and the spermatozoa $\left(r=0.813^{* *}\right)$

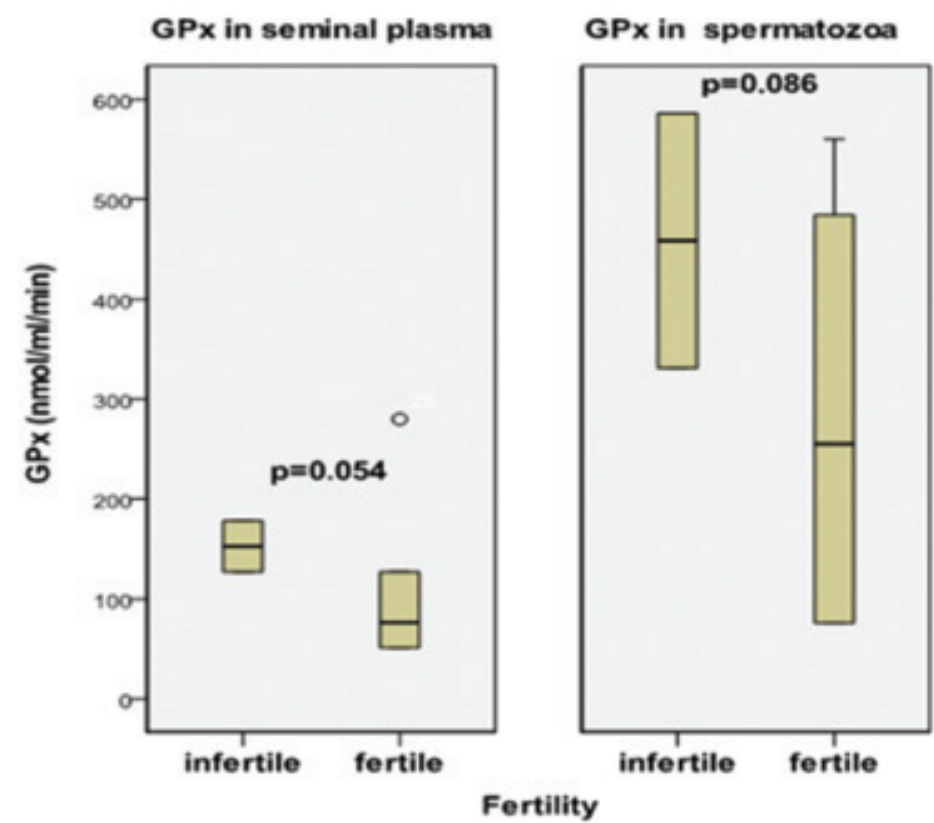

Figure 4. Glutathione peroxidase activity $(\mathrm{nmol} / \mathrm{ml} / \mathrm{min})$ in seminal plasma and spermatozoa of fertile and infertile men

were close to the significant values $(\mathrm{p}=0.057)$. However, the levels were once again higher in the fertile group $(0.56 \pm 0.3$ compared to $0.4, \pm$ $0.29 \mu \mathrm{M}$ in the infertile group). (Figure 2). We found a positive correlation between the levels of GSH in seminal plasma and GSH in the spermatozoa of all subjects $r=0.813^{* *}$ ) (Figure $3)$.

\section{Glutathione peroxidase}

The results from the GPx measurement are presented in Figure 4. No significant differences in the enzyme activity in the investigated subject groups were discovered. An interesting deviation from normal was the higher enzyme activity in spermatozoa registered in the fertile group. The significant coefficient was very close to $0.05-$ 0.054 for the seminal plasma and 0.086 for the spermatozoa.

\section{Discussion}

In this research, we measured and compared 
the activity of the GPx enzyme and the levels of its substrate GSH in the seminal plasma and the spermatozoa of patients with unexplained infertility with those of fertile men. All samples we collected underwent conventional semen analysis. This made it possible to select suitable participants in the research. The investigation of the subjects from both groups showed that the analyzed characteristics are higher than the minimum values defined by the WHO in 2010. All participants were defined as normozoospermic. They were separated based on achieved pregnancy from regular and unprotected sexual contacts for a calendar year. The groups were formed in such a way because of a widespread opinion that the essential seminal characteristics are not a guarantee or standard for screening and men infertility identification [16].

Free radicals and antioxidative systems have been a subject of investigation for many years now. Nevertheless, their influence on male fertility is still underestimated. The optimal balance between free radicals production and elimination is what spermatozoa need for achieving conception and normal pregnancy. Cells in a biological environment, such as the ejaculate, help the oxidative homeostasis through different systems. Low levels of ROS are necessary for the normal function of the cells [17]. The control depends on antioxidants. Glutathione is one of the most important natural antioxidants, and it helps the activity of other antioxidants. It can be found in both male and female gametes, and its level depends on the localization [18]. Glutathione is vital for the stability of the spermatozoon's midpiece $[19,20]$. Glutathione also protects the plasma membrane of the lipid peroxidation, eliminates the peroxides, and prevents the formation of oxygen respectively of superoxide anions. Most scientific publications claim that low glutathione levels can affect male fertility potential [21]. If the sperm of infertile men with varicocele or inflammation interacts with glutathione, an increase in the sperm quality is spotted (Lenzi et al., 1993) [22].

Despite all the conducted researches in this field, the data is still controversial. There are no significant differences between the glutathione levels in the investigated groups of men with different fertility potential. In the literature, one can find data of lower glutathione levels found in men with azoospermia, asthenozoospermia, oligospermia, and teratozoospermia, in comparison with normozoospermic men $[23,24]$. There is also information about cases when high levels of glutathione are connected with infertility [25]. However, publications comparing glutathione levels in sperm and seminal plasma in unexplained fertility men are very few [25]. Unlike Ebisch et al., our results confirm the role of glutathione in male fertility. We measured significantly higher levels of this thiol in the seminal plasma of fertile men than in the plasma of infertile men. The intracellular glutathione levels were higher in the fertile men, too, but the differences were not that significant. Despite this fact, we think our results definitely demonstrate the role of glutathione in male fertility.

Human sperm depends on the system glutathione peroxidase/ glutathione reductase/ glutathione as a substrate for the inactivation of ROS. In search for differences between the GPx activity in fertile men and in men with unexplained fertility, we measured the activity of this enzyme in both seminal plasma and spermatozoa. The differences we found between the two groups were not significant. However, unlike most researches reported in the literature, we discovered a higher activity of the enzyme in the plasma and spermatozoa in the infertile group, though the difference was not significant $(p=0.054$ for the seminal plasma and $p=0.086$ for spermatozoa).

Of all the eight enzymes from the peroxidase family, GPx4 or PHGPx (phospholipid hydroperoxidase) is the most important for male sperm. It exists in three isoforms - cytosolic (cGPx) in every cell, mitochondrial (mGPx), and nuclear in the spermatozoa (snGPx) [26]. The mitochondrial isoform represents $50 \%$ of the proteins in the midpiece of a spermatozoon and is important for forming mitochondrial components [20]. The need for normal functioning mGPx4 for male fertility has been proven in mice and humans. Mice with a knockdown gene for mGPx 4 are healthy, but male mice are infertile, and their spermatozoa have many anomalies concerning morphology and motility [27].

As far as the nuclear isoform snGPx is concerned, its significance is not directly proportional to male fertility. This isoform plays a role during spermatogenesis and is connected 
with the oxidation of thiol groups of protamines by the hydroperoxides. However, mice with knockdown genes for snGPx4 are fertile, which means that the chromatin formation is compensated by other protein systems [28], and if the process is not completed in the testes, it continues in the epididymis under the influence of the seminal GPx. Most publications published associate infertility with low GPx levels. The activity of GPx tends to be lower in the samples of patients with asthenozoospermia, oligozoospermia, and teratozoospermia compared to samples of normozoospermic men [29]. There are reports in the literature about the higher activity of the enzyme registered in patients with oligozoospermia [30,31,32]. Giannattasio et al. inform of higher GPx activity in the plasma of fertile men than in men with idiopathic infertility [33]. According to them, almost $100 \%$ of the activity is due to GPx 3 despite the widely spread opinion that this peroxidase is not typical for seminal plasma. However, there are no scientific publications that compare the enzyme's activity in fertile men and in those with unexplained infertility, and researches of GPx in human spermatozoa are very few. The logical reason for that is the small quality of the cytoplasm. In our study, the activity registered by the Cayman Chemicals test was at its highest level in spermatozoa in the infertile group. The possible reason for this finding, which is different from the conventional opinion, is that it is the increase in the activity of GPx itself that could be the reason for the low fertilization rate of the spermatozoa in the patient's group. As a scavenger of hydrogen peroxide, GPx decreases the concentration of hydrogen peroxide to an extent to interfere with the process of optimal capacitation at the moment of fertilization.

It is noteworthy that the peroxidase activity in spermatozoa was more clearly defined than that in the seminal plasma. The higher enzyme activity in the spermatozoa should not be due to the absorbed seminal plasma or the enzyme molecules on the cell/spermatozoon surface. The spermatozoa underwent ultrasound treatment to rupture their membranes and measure their intracellular antioxidative activity of GPx. The registered activity is probably due to the nuclear $(\mathrm{snGPx} 4)$ and mitochondrial (mGPx) glutathione peroxidase [26].

\section{Conclusion}

The results from our investigation confirmed the connection between low glutathione levels in seminal fluid and male fertility. They cast doubt on the widespread opinion that the activity of the antioxidative glutathione peroxidase is lowered in the sperm of infertile men. Investigations like this show it is necessary to define the levels of pro- and antioxidants when diagnosing and treating unexplained infertility in men.

\section{Acknowledgements}

This work was supported by the project No D3/2017 of Medical University - Pleven.

\section{References}

1. Practice Committee of the American Society for Reproductive Medicine. Definitions of Infertility and Recurrent Pregnancy Loss: a Committee Opinion. Fertility Sterilty. 2013;99:63

2. Patel R, Rinker L, Peng J, Chilian WM. Reactive Oxygen Species: The Good and the Bad. In: Filip C, Albu E, editors. Reactive Oxygen Species (ROS) in Living Cells. 2018;23-41.

3. Alahmar AT. Role of Oxidative Stress in Male Infertility: An Updated Review. J Hum Reprod Sci. 2019 Jan-Mar;12(1):4-18.

4. Snezhkina AV, Kudryavtseva AV. Kardymon OL, Savvateeva MV, Melnikova NV, Krasnov GS, et al. ROS Generation and Antioxidant Defense Systems in Normal and Malignant Cells. Oxidative Medicine and Cellular Longevity. 2019;Apr 5:17.

5. Nenkova G, Alexandrova A. A review: Oxidative stress and its role in reproduction. Advances in Bioscience and Biotechnology. 2013;4:37-43.

6. Gudeloglu A, Brahmbhatt J. Definitions and Epidemiology of Unexplained Male Infertility. In: Schattman GL, Esteves CS, editors. Unexplained Infertility. New York: Andrology and Human Reproduction Clinic. 2015:7-12.

7. Hamada A, Esteves SC, Nizza M, Agarwal A. Unexplained male infertility: diagnosis and management. Int Braz J Urol. 2012 Oct;38(5):576-94.

8. Agarwal A, Saleh RA, Bedaiwy MA: Role of reactive oxygen species in the pathophysiology of human reproduction. Fertil Steril. 2003 Apr;79(4):829-43.

9. Said TM, Agarwal A, Sharma RK, Thomas AJ, Sikka SC. Impact of sperm morphology on DNA 
damage caused by oxidative stress induced by $\beta$-nicotinamide adenine dinucleotide phosphate. Fertility and Sterility. 2005 Jan;83(1):95-103.

10. Jones R, Mann T, Sherins R: Peroxidative breakdown of phospholipids in human spermatozoa, spermicidal properties of fatty acid peroxides, and protective action of seminal plasma. Fertility and Sterility. 1979 May;31(5):531-7

11. Hidalgo DM, Bragado MJ, Batista AR, Oliveira PF, Alves MG. Antioxidants and Male Fertility: from Molecular Studies to Clinical Evidence. Antioxidants 2019 Apr 5;8(4):89.

12. O'Flaherty C. The Enzymatic Antioxidant System of Human Spermatozoa. Advances in Andrology. 2014:1-15.

13. Drevet JR. The antioxidant glutathione peroxidase family and spermatozoa: A complex story. Molecular and Cellular Endocrinology. 2006 May 16;250(1-2):70-9.

14. Agrawal YP, Vanha-Perttula T. Glutathione, Lglutamic acid and gamma-glutamyl transpeptidase in the bull reproductive tissues. Int J Androl. 1988 Apr;11(2):123-31.

15. Griveau JF, Le Lannou D. Reactive oxygen species and human spermatozoa: physiology and pathology. Int J Androl. 1997;20:61-9.

16. Mayorga-Torres BJ, Camargo M, Cadavid AP, Plessis SS, Cardona Maya WD. Are oxidative stress markers associated with unexplained male infertility? Andrologia 2017:49.

17. O'Flaherty C, de Lamirande E, Gagnon C. Positive role of reactive oxygen species in mammalian sperm capacitation: triggering and modulation of phosphorylation events. Free Radical Biol Med. 2006 Aug 15;41(4):528-40.

18. Luberda $Z$. The role of glutathione in mammalian gametes. Reprod Biol. 2005 Mar;5(1):5-17.

19. Villa NA, Castaño D, Duque PC, Ceballos A. Glutathione Peroxidase and Superoxide Dismutase activities in blood and seminal plasma in colombian stallions. Rev Colomb Cienc Pecu. 2012;25:64-70.

20. Ursini, F, Heim, S, Kiess, M, Maiorino, M, Roveri, A, Wissing J, et al. Dual function of the selenoprotein PHGPx during sperm maturation. Science. 1999;285(5432):1393-6.

21. Chaudhari AR, Piyali D, Ramji S. Study of oxidative stress and reduced glutathione levels in seminal plasma of human subjects with different fertility potential. Biomedical Research [Internet]. 2008;19(3):207-10.

22. Lenzi A, Culasso F, Gandini L, Lombardo F, Dondero F. Placebo-controlled, doubleblind, cross-over trial of glutathione therapy in male infertility. Human Reproduction. 1993;8(10):1657-662.
23. Ochsendorf FR, Buhl R, Bastlein A, Beschmann H. Glutathione in Spermatozoa \& Seminal Plasma of infertile men. Hum Reprod. 1998;13:353-9.

24. Atig F, Raffa M, Habib BA, Kerkeni A, Saad A, Ajina M. Impact of seminal trace element and glutathione levels on semen quality of Tunisian infertile men. BMC Urol. 2012;12:6.

25. Ebisch IMW, Peters WHM, Thomas CMG, Wetzels AMM, Peer PGM, Steegers-Theunissen RPM. Homocysteine, glutathione and related thiols affect fertility parameters in the (sub) fertile couple. Human Reproduction. 2006 Jul;21(7):1725-33.

26. Brigelius-Flohe R, Aumann KD, Blocker H, Gross G, Kiess M, Kloppel KD, et al. Phospholipid-hydroperoxide glutathione peroxidase. Genomic DNA, cDNA, and deduced amino acid sequence. J. Biol. Chem. 1994 Mar 11;269(10):7342-8.

27. Förster H, Boersma A, Seiler A, Wehnes H, Sinowatz F, Neumüller C, Deutsch, MJ, Walch A, Hrabe' deAngelis M, Wurst W, Ursini F, Roveri A, Maleszewski M, Maiorino M, Conrad M. Mitochondrial glutathioneperoxidase 4 disruption causes male infertility. FASEB J.2009;23:3233-42.

28. Conrad M, Moreno SG, Sinowatz, F, Ursini F, Kolle S, Roveri A, et. al. The nuclear form of phospholipid hydroperoxide glutathione peroxidase is a protein thiol peroxidase contributing to sperm chromatin stability. Mol. Cell. Biol. 2005 Sept;25(17):7637-44.

29. Huang C, Cao X, Pang D, Li C, Luo Q, Zou Y, et al. Is male infertility associated with increased oxidative stress in seminal plasma? A-meta analysis. 2018 May 11;9(36):24494-513.

30. Türk S, Mändar R, Mahlapuu R, Viitak A, Punab M, Kullisaar T. Male infertility: Decreased levels of selenium, zinc and antioxidants. J. Trace Elem. Med. Biol. 2014 Apr;28(2):179-85.

31. Tavilani H, Goodarzi MT, Vaisi-Raygani A, Salimi S, Hassanzadeh T. Activity of antioxidant enzymes in seminal plasma and their relationship with lipid peroxidation of spermatozoa. Int Braz J Urol. 2008 Aug;34(4):485-91.

32. Khalil AA. Oxidative stress induces idiopathic infertility in Egyptian males. African Journal of Biotechnology. 2012 Jan;11(6):1516-22. 